\title{
On studying relations between time series in climatology
}

\author{
V. Privalsky ${ }^{1, a}$ \\ ${ }^{1}$ Space Dynamics Laboratory, Logan, Utah, USA \\ a retired \\ Correspondence to: V. Privalsky (vprivalsky@gmail.com) \\ Received: 18 February 2015 - Published in Earth Syst. Dynam. Discuss.: 01 April 2015 \\ Revised: 26 May 2015 - Accepted: 05 June 2015 - Published: 24 June 2015
}

\begin{abstract}
Relationships between time series are often studied on the basis of cross-correlation coefficients and regression equations. This approach is generally incorrect for time series, irrespective of the cross-correlation coefficient value, because relations between time series are frequency-dependent. Multivariate time series should be analyzed in both time and frequency domains, including fitting a parametric (preferably, autoregressive) stochastic difference equation to the time series and then calculating functions of frequency such as spectra and coherent spectra, coherences, and frequency response functions. The example with a bivariate time series "Atlantic Multidecadal Oscillation (AMO) - sea surface temperature in Niño area 3.4 (SST3.4)" proves that even when the cross correlation is low, the time series' components can be closely related to each other. A full time and frequency domain description of this bivariate time series is given. The AMO-SST3.4 time series is shown to form a closed-feedback loop system with a 2-year memory. The coherence between AMO and SST3.4 is statistically significant at intermediate frequencies where the coherent spectra amount up to $55 \%$ of the total spectral densities. The gain factors are also described. Some recommendations are offered regarding time series analysis in climatology.
\end{abstract}

\section{Introduction}

Studying relations between time series on the basis of observations is a common task in all branches of Earth sciences. Normally, it requires getting quantitative answers to the following questions:

- What is the optimal time-domain stochastic model for a given multivariate time series?

- Which components of the time series could be regarded as inputs and outputs of a respective hydrometeorological system?

- Is there any interaction between the inputs and the outputs (are there any closed-feedback loops within the system)?

- What are the statistical properties of the multivariate time series in the time and frequency domains?
In this article, we will apply the methods first developed in theory of information (Gelfand and Yaglom, 1956), time series analysis (Bendat and Piersol, 1966; Box and Jenkins, 1970), econometrics (Granger and Hatanaka, 1964; Granger, 1969), and in geophysics (Robinson, 1967; Robinson and Treitel, 1980) to study relations between multivariate time series of climatic data. The goal is to describe time series in the time and frequency domains, including climatic teleconnections that can hardly be found within the correlation/regression approach. Although methods of multivariate time series analysis are also described, mostly in the frequency domain, in climatology (von Storch and Zviers, 1999) and oceanography (Emery and Thomson, 2004), they are rarely applied in both time and frequency domains (e.g., Privalsky, 1988; Privalsky and Jensen, 1995) or just in the frequency domain (e.g., Oladipo, 1987; Schneider and Schönwiese, 1989; Ghanbari et al., 2009; Park and Dusek, 2013). 


\section{Elements of multivariate time series analysis}

Note first that the linear correlation/regression approach as a means of studying relations between scalar time series, including teleconnections within the climatic system, is generally inapplicable to time series analysis. The simplest example given in Privalsky and Jensen (1995) and repeated in Emery and Thomson (2004) is a zero cross-correlation coefficient between two white noise sequences, one of which is obtained by applying a shift operator to the other. A low correlation coefficient may occur between any time series related to each other through more complicated but still strictly linear transformations. In particular, it can be a time series and its first difference, or any autoregressive-movingaverage (ARMA) time series and its innovation sequence, or the time series at the input and output of a linear filter. The general statement is that if a time series is obtained from another time series through a linear inertial transformation, the correlation coefficient between them will not be equal to 1 in spite of the strictly linear dependence between them.

Relations between two time series (say, $A$ and $B$ ), which are not mutually independent, always correspond to one of the following three situations: $A$ affects $B$ but not vice versa, $B$ affects $A$ but not vice versa, $A$ and $B$ affect each other (interaction). Again, the correlation/regression approach does not allow one to determine what the actual situation is. It can be done within the framework of the time- and frequencydomain analysis of multivariate time series.

The linear regression equation $B=\alpha A+\varepsilon$, where $\alpha$ is a constant and $\varepsilon$ is a white noise sequence (regression error), means that the spectra $s_{A}(f), s_{B}(f)$ of the time series $A$ and $B$ are identically shaped because $s_{A}(f)=\alpha^{2} s_{B}(f)+$ const (where $f$ is frequency and the constant depends upon the regression error variance). This result is irrelevant when $A$ and $B$ are time-invariant random variables but if $A$ and $B$ are time series, it puts an unnecessary limitation upon their properties. In the general case, the shapes of the spectra are not identical, which would mean that $\varepsilon$ is not white noise thus making the regression equation inadequate. This is another reason why both the cross-correlation coefficient and respective linear regression equation cannot describe relations between time series.

The problem is solved if one uses methods of time series analysis including simultaneous description of multivariate time series in the time and frequency domains. It means fitting a stochastic difference equation to the time series, analyzing its properties in the time domain and then calculating and analyzing functions that describe the time series in the frequency domain. For a number of considerations (see below), the approach used here will be limited to the autoregressive (AR) case. Also, we will only be regarding the bivariate case. The extension to higher dimensions is rather simple (e.g., Bendat and Piersol, 1966; Robinson, 1967) and will briefly be described at the end of this section.
Let the bivariate time series $\boldsymbol{x}_{n}=\left[x_{1, n}, x_{2, n}\right]^{\prime}$ be a (zero mean) sample record of an ergodic discrete-time random process; here $n=1, \ldots, N$ is the dimensionless argument, $N \Delta t$ time series length in time units $\Delta t$, and the strike means matrix transposition. The sampling interval $\Delta t$ is supposed here to be 1 year.

In the time domain, the time series is described with a stochastic difference equation

$\boldsymbol{x}_{n}=\sum_{j=1}^{p} \boldsymbol{\Phi}_{j} \boldsymbol{x}_{n-j}+\boldsymbol{a}_{n}$,

where $p<\infty$ is the order of autoregression,

$\boldsymbol{\Phi}_{j}=\left[\begin{array}{cc}\varphi_{11}^{(j)} & \varphi_{12}^{(j)} \\ \varphi_{21}^{(j)} & \varphi_{22}^{(j)}\end{array}\right]$

are matrix AR coefficients, and $\boldsymbol{a}_{n}=\left[a_{1, n}, a_{2, n}\right]^{\prime}$ is a bivariate innovation sequence (white noise) with a covariance matrix

$\mathbf{R}_{a}=\left[\begin{array}{ll}R_{11} & R_{12} \\ R_{21} & R_{22}\end{array}\right]$.

The order $p$ of an optimal AR model that agrees with the observed time series $\boldsymbol{x}_{n}$ must be chosen on the basis of quantitative statistical considerations. Probably, the most efficient way to select an optimal order is to use order-selection criteria such as Akaike's AIC, Schwarz-Rissanen's BIC, Parzen's CAT, and Hannan-Quinn's $\varphi$ (e.g., Box et al., 2008; Parzen, 1977; Hannan and Quinn, 1979).

Properties of the time series $\boldsymbol{x}_{n}$ in the frequency domain are defined with the spectral matrix

$\mathbf{s}(f)=\left[\begin{array}{ll}s_{11}(f) & s_{12}(f) \\ s_{21}(f) & s_{22}(f)\end{array}\right]$,

which is obtained through a Fourier transform of stochastic difference Eq. (1) fitted to the time series $\boldsymbol{x}_{n}$. Here $f$ is frequency in cycles per sampling interval (in our case, $\left.\mathrm{yr}^{-1}\right), s_{11}(f), s_{22}(f)$ are spectra and $s_{12}(f), s_{21}(f)$ complexconjugated cross-spectra of the time series components $x_{1, n}$, and $x_{2, n}$. In particular, the coherence function

$\gamma_{12}(f)=\frac{\left|s_{12}(f)\right|}{\left[s_{11}(f) s_{22}(f)\right]^{1 / 2}}$

describes the linear dependence between the time series components $x_{1, n}$ and $x_{2, n}$ in the frequency domain. It can be thought of as a frequency-dependent "correlation coefficient" between the components of a bivariate time series. It is the coherence function $\gamma_{12}(f)$ (and not the cross-correlation coefficient) that describes the degree of linear dependence between two scalar time series. Values of $\gamma_{12}(f)$ satisfy the condition $0 \leq \gamma_{12}(f) \leq 1$. 
The importance of the coherence function in time series analysis and modeling is illustrated with the following property. If the components of an ergodic bivariate time series present processes at the input and output of any linear timeinvariant system, the coherence between them will be equal to 1 at all frequencies where the spectral density is not too close to zero.

The spectral matrix (Eq. 4) describes a linear stochastic system with the time series $x_{2, n}$ and $x_{1, n}$ as the system's input and output, respectively. The coherent spectral density, or coherent spectrum $G_{12}(f)=\gamma_{12}^{2}(f) s_{11}(f)$ defines the part of the output spectrum $s_{11}(f)$ that can be explained by the linear dependence between $x_{1, n}$ and $x_{2, n}$. The coherent spectrum $G_{21}(f)=\gamma_{12}^{2}(f) s_{22}(f)$ describes the share of the spectrum $s_{22}(f)$ defined by the contribution of $x_{1, n}$ to $x_{2, n}$. Finally, the complex-valued frequency response function $H_{12}(f)=s_{12}(f) / s_{22}(f)$ shows in what manner the spectral energy $s_{22}(f)$ of the input $x_{2, t}$ is transformed into the spectral energy $s_{11}(f)$ of the output $x_{1, t}$ as well as the phase difference between them (the gain factor $\left|H_{12}(f)\right|$ and phase factor $\phi_{12}(f)$, respectively). The spectral characteristics calculated on the basis of Gaussian AR models with properly selected autoregressive orders satisfy the requirements of the maximum entropy method in spectral analysis. This is one of the reasons for selecting the AR modeling.

In the general case of an $M$-variate time series $\boldsymbol{x}_{n}=\left[x_{1, n}, \ldots, x_{M, n}\right]^{\prime}$, the time-domain model is still given by Eq. (1) with the matrix AR coefficients

$\boldsymbol{\Phi}_{j}=\left[\begin{array}{cccc}\varphi_{11}^{(j)} & \varphi_{12}^{(j)} & \ldots & \varphi_{1 M}^{(j)} \\ \varphi_{21}^{(j)} & \varphi_{22}^{(j)} & \ldots & \varphi_{2 M}^{(j)} \\ \vdots & \vdots & & \vdots \\ \varphi_{M 1}^{(j)} & \varphi_{M 2}^{(j)} & \ldots & \varphi_{M M}^{(j)}\end{array}\right]$

and with the innovation sequence $\boldsymbol{a}_{n}=\left[a_{1, n}, \ldots, a_{M, n}\right]^{\prime}$. Its covariance matrix

$\mathbf{R}_{a}=\left[\begin{array}{cccc}R_{11} & R_{12} & \ldots & R_{1 M} \\ R_{21} & R_{22} & \ldots & R_{2 M} \\ \vdots & \vdots & & \vdots \\ R_{M 1} & R_{M 2} & \ldots & R_{M M}\end{array}\right]$.

Consequently, the spectral matrix (Eq. 4) changes to

$\boldsymbol{s}(f)=\left[\begin{array}{cccc}s_{11}(f) & s_{12}(f) & \ldots & s_{1 M}(f) \\ s_{21}(f) & s_{22}(f) & \ldots & s_{2 M}(f) \\ \vdots & \vdots & & \vdots \\ s_{M 1}(f) & s_{M 2}(f) & \ldots & s_{M M}(f)\end{array}\right]$,

where $s_{i j}(f)$ are spectral (if $i=j$ ) and cross-spectral (if $i \neq j$ ) densities, respectively, of the time series components $x_{i, n}, i=1, \ldots, M$. The elements of the spectral matrix (Eq. 8) are used to calculate spectra, multiple and partial coherence functions, multiple and partial coherent spectra, and $M-1$ frequency response functions (see Bendat and Piersol, 2010). The spectral matrix (Eq. 8) is Hermitian.

It should be noted that if the multivariate time series is long (by orders of magnitude longer than the largest timescale of interest) and if the spectra of its components are intricate, the above-described approach may not be the best, especially in the time domain - because of the high order of the optimal stochastic difference equation. In such cases the analysis may have to be limited to a frequency domain description of the time series by using a parametric or nonparametric (e.g., Percival and Walden, 1993; Bendat and Piersol, 2010) approach. However, this can hardly happen if one is interested in properties of time series at climatic timescales.

\section{An example of bivariate time series analysis}

The example with actual climatic data given below proves that the components of a bivariate time series can be connected to each other in spite of the fact that the crosscorrelation coefficient between them is low. It also provides a simultaneous description of a climatic system in both time and frequency domains. The case with a high crosscorrelation coefficient between the components of the ENSO system (Southern Oscillation Index and SST variations in the Niño area 3.4) has been treated in detail in Privalsky and Muzylev (2013) where it was shown, in particular, that both time series are close to white noise, interact with each other mostly through the innovation sequence, and that the coherence function, coherent spectra, and the frequency response functions between SOI and SST are frequency-dependent.

The El Niño-Southern Oscillation (ENSO) system is believed to affect many phenomena in the Earth's climate (e.g., Philander, 1989). We will construct an autoregressive model of the bivariate time series $\boldsymbol{x}_{n}=\left[x_{1, n}, x_{2, n}\right]^{\prime}$, which consists, respectively, of the annual Atlantic Multidecadal Oscillation (AMO) time series $x_{1, n}$ (Kaplan SST V2 data provided by the NOAA/OAR/ESRL PSD, Boulder, Colorado, USA, see Enfield et al., 2001) and sea surface temperature SST3.4 - an oceanic component of the ENSO system $\left(x_{2, n}\right)$. The SST3.4 data are calculated from the HadSST3 file at the University of East Anglia site (see Morice et al., 2012), for the same time interval from 1876 through 2014 (Fig. 1a).

As seen from the figure, the two components behave in a different manner: AMO contains much stronger lowfrequency variations than SST3.4. The correlation between AMO and SST3.4 (Fig. 1b) is very low, with the crosscorrelation coefficient 0.06 and the maximum absolute values of the cross-correlation function below 0.26 . With the cross-correlation-based approach that prevails in climatology, the conclusion would have to be that the two scalar time series are either not related to each other at all or that the connection between them is very weak. And it would not be correct. 

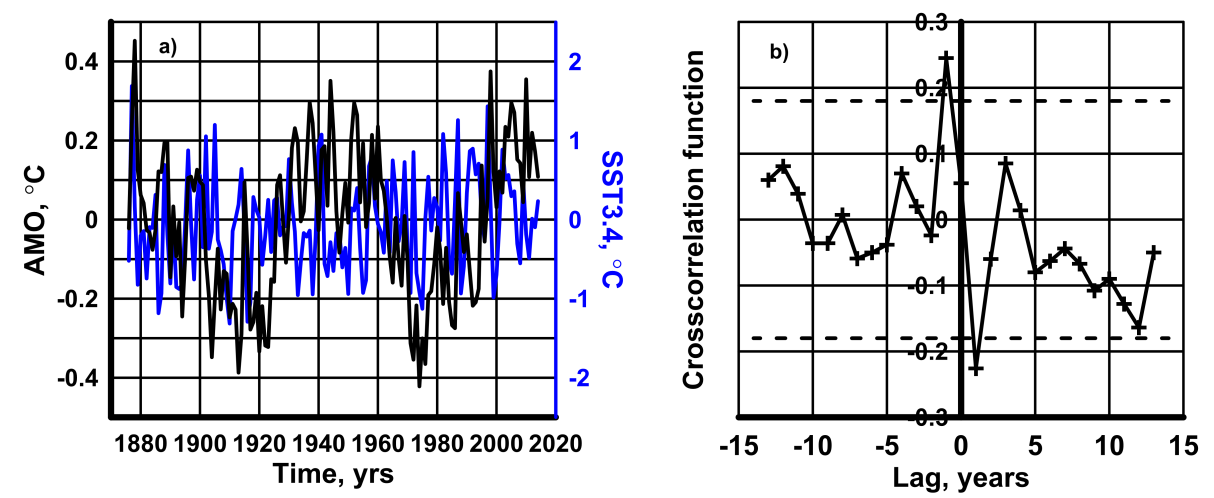

Figure 1. Observed AMO and SST3.4, 1876-2014 (a) and AMO-SST3.4 cross-correlation function estimate (b); the dashed lines show approximate $90 \%$ confidence bounds.
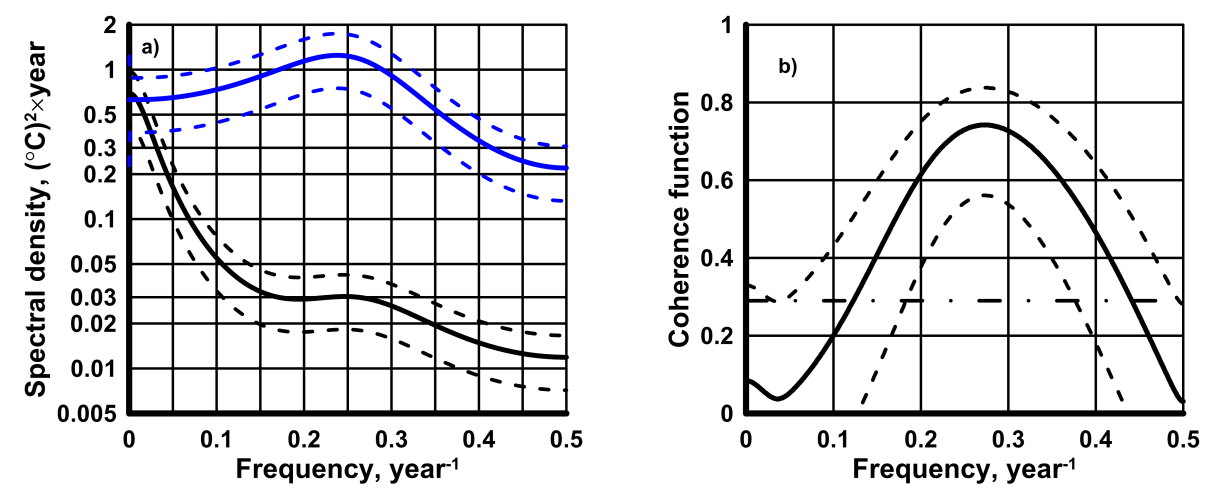

Figure 2. (a) Spectra of AMO (black lines) and SST3.4 (blue lines) with approximate $90 \%$ confidence bounds (dashed lines); (b) coherence function AMO-SST3.4 (solid line) with approximate $90 \%$ confidence bounds (dashed lines). The dot-dashed line shows the approximate upper $90 \%$ confidence bound for the true zero coherence. For confidence bounds see Privalsky et al. (1987) and Appendix below.

Consider first the time-domain properties. All four abovementioned order selection criteria selected the same order $p=2$. The respective $\mathrm{AR}(2)$ model is

$$
\begin{aligned}
x_{1, n} & \approx 0.59 x_{1, n-1}+0.09 x_{2, n-1}+0.21 x_{1, n-2} \\
& -0.07 x_{2, n-2}+a_{1, n} \\
x_{2, n} & \approx-1.28 x_{1, n-1}+0.29 x_{2, n-1}+1.00 x_{1, n-2} \\
& -0.23 x_{2, n-2}+a_{2, n}
\end{aligned}
$$

with the innovation covariance matrix

$\mathbf{R}_{a}=\left[\begin{array}{ll}0.014 & 0.007 \\ 0.007 & 0.290\end{array}\right]$

All coefficients in Eq. (9) are statistically significant at a confidence level of 0.95 .

Obviously, Eq. (9) describes a closed-feedback loop system: AMO $\left(x_{1, n}\right)$ depends upon two of its previous values and upon two previous values of SST3.4 $\left(x_{2, n}\right)$ and SST3.4 depends upon two previous values of both SST3.4 and AMO. The eigenfrequency of this system was found to be $0.24 \mathrm{yr}^{-1}$ so that a respective period of about 4 years is the time required to close the system's feedback loop.

The stochastic difference (Eq. 9) and the innovation sequence covariance matrix (Eq. 10) allow one to understand how much of the variances of AMO and SST3.4 can be explained by the "deterministic" components of the model (Eq. 9) that describes the dependence of $x_{1, n}$ (AMO) and $x_{2, n}$ (SST3.4) upon their own past values and upon past values of the other time series. The variance of $\mathrm{AMO} \sigma_{1}^{2} \approx 0.035\left({ }^{\circ} \mathrm{C}\right)^{2}$ while, according to Eq. (10), the variance $R_{11}$ of the innovation sequence $a_{1, n}$ is $0.014\left({ }^{\circ} \mathrm{C}\right)^{2}$. Therefore, the part of the AMO variance $\sigma_{1}^{2}$ which cannot be explained by the dependence of the time series upon their past behavior is $0.014 / 0.035 \approx 0.4$. Thus, Eq. (9) allows one to explain about $60 \%$ of the AMO variance by its dependence upon its own past values and upon the past values of SST3.4.

The SST3.4 variance $\sigma_{2}^{2} \approx 0.38\left({ }^{\circ} \mathrm{C}\right)^{2}$ while the variance $R_{22}$ of the innovation sequence $a_{2, n}$ is $0.29\left({ }^{\circ} \mathrm{C}\right)^{2}$. Thus, the unexplainable part of the SST3.4 variance amounts to about $75 \%$ of the SST3.4 variance. The results for AMO and SST3.4 differ because AMO and SST3.4 have very dissimilar spectra (Fig. 2a). 

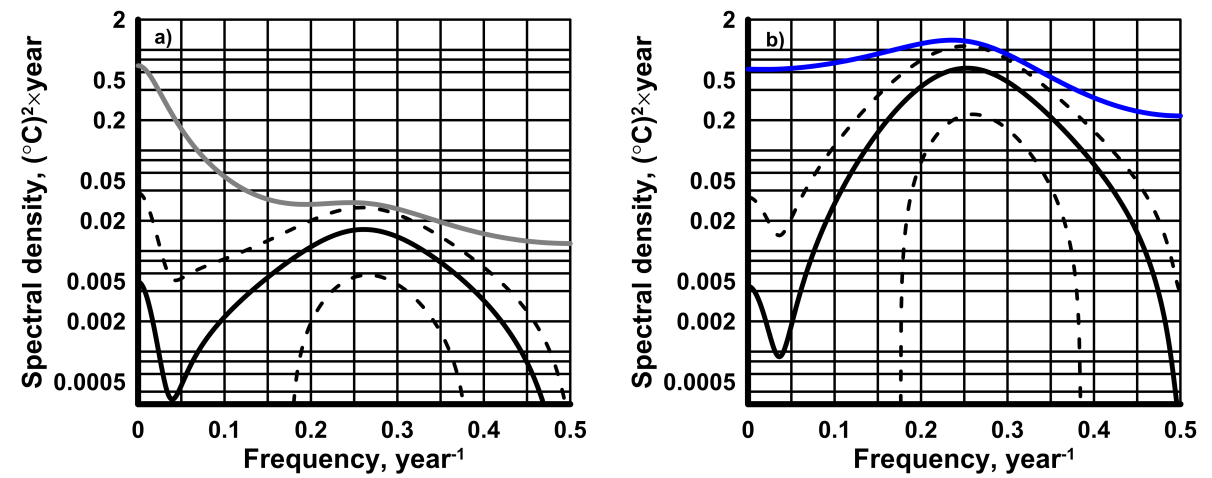

Figure 3. Coherent spectra: contribution of SST3.4 to the AMO spectrum (a), and contribution of AMO to the SST3.4 spectrum (b). Dashed, grey, and blue lines show approximate $90 \%$ confidence bounds and AMO and SST3.4 spectra, respectively.

Both AMO and SST3.4 time series can be regarded as Gaussian so that their autoregressive spectral estimates satisfy the requirements of the maximum entropy spectral analysis. The AMO spectrum $s_{11}(f)$ quickly decreases with frequency, which is characteristic of spatially averaged climatic processes. Such behavior of the spectrum means that AMO is strongly dependent on its past values. The SST3.4 spectrum $s_{22}(f)$ is not monotonic, has a wide maximum at intermediate frequencies and, in general, does not differ much from a white noise spectrum. The dependence on its past values is weak.

Though the cross-correlation coefficient between AMO and SST3.4 is very low, the maximum entropy estimate of coherence $\gamma_{12}(f)$ shown in Fig. $2 \mathrm{~b}$ is statistically significant everywhere except at the frequencies below $0.13 \mathrm{yr}^{-1}$ and above $0.43 \mathrm{yr}^{-1}$. It is bell-shaped and exceeds 0.6 within the frequency band from 0.20 to $0.36 \mathrm{yr}^{-1}$, that is, roughly, at timescales between 3 and 5 years. Its maximum value is 0.74 at $f \approx 0.27 \mathrm{yr}^{-1}$. This behavior of the coherence function means that the components of this bivariate time series contribute to each other up to about $55 \%$ of the spectral density at intermediate frequencies.

The coherence between AMO and SST3.4 is weak at the low-frequency end, where AMO's spectral energy is much higher than elsewhere. The high coherence occurs at intermediate frequencies where the spectral density of AMO is much lower. The strong dependence of AMO on its past values and the relative closeness of the SST3.4 spectrum to a constant seem to be the reasons why the stochastic model (Eq. 9) can explain so much of the total AMO variance and less of the total SST3.4 variance.

The contribution of SST3.4 to the AMO spectrum is $G_{12}(f)=\gamma_{12}^{2}(f) s_{11}(f)$, where $s_{11}(f)$ is the AMO spectrum (Fig. 3a). Respective contribution of AMO to SST3.4 shown in Fig. $3 \mathrm{~b}$ is $G_{21}(f)=\gamma_{12}^{2} s_{22}(f)$. These coherent spectrum estimates are statistically significant within the frequency band from 0.18 to $0.38 \mathrm{yr}^{-1}$, where they amount to $25-$ $55 \%$ of the spectral densities $s_{11}(f)$ and $s_{22}(f)$. This is a substantial contribution but it occurs within the frequency band where the spectral density of AMO is at least an order of magnitude smaller than at lower frequencies where the coherence between AMO and SST3.4 is not significant. The cross-correlation coefficient that "integrates" the coherence function is small in spite of the relatively close connection between the two processes at moderate frequencies for at least two reasons: the complex structure of the interdependence between the time series components expressed by Eqs. (9) and (10) which cannot be described with a linear regression equation and the low absolute contribution of SST3.4 to the AMO variance.

This example also shows that using proper methods of analysis allows one to avoid filtering of time series in order to suppress "noise". Indeed, though the low-frequency variations dominate the spectrum of $\mathrm{AMO}$, the coherence function has revealed the "signal" - a teleconnection between AMO and SST3.4 at intermediate frequencies where the AMO spectrum is low. This is another useful property of autoregressive time- and frequency-domain models.

Our frequency-domain results generally agree with the earlier results by Park and Dusek (2013) regarding the connection between AMO and the Multivariate ENSO Index (MEI) at intermediate frequencies. The authors used a nonparametric spectral estimation-singular spectrum analysis keeping 10 first empirical orthogonal functions that cover slightly over $75 \%$ of the time series total variances. At frequencies above $0.15 \mathrm{yr}^{-1}$, our estimate of coherence is quite similar to the coherence estimate in Park and Dusek (2013). However, the authors did not estimate the frequency response function (FRF) because, according to them, "a physically based transfer function is likely [to be] nonlinear". Actually, a nonlinear theoretical model of the FRF between AMO and SST3.4 time series would have been in disagreement with observations because both time series are Gaussian. At frequencies from 0.1 to $0.4 \mathrm{yr}^{-1}$, the gain factors of the empirical FRFs AMO-SST3.4 and SST3.4-AMO (Fig. 4) behave in the same manner as the coherent spectra shown in Fig. 3 and their values at intermediate frequencies amount to approximately 0.1 and 4.0 , respectively. The coherent spectra 

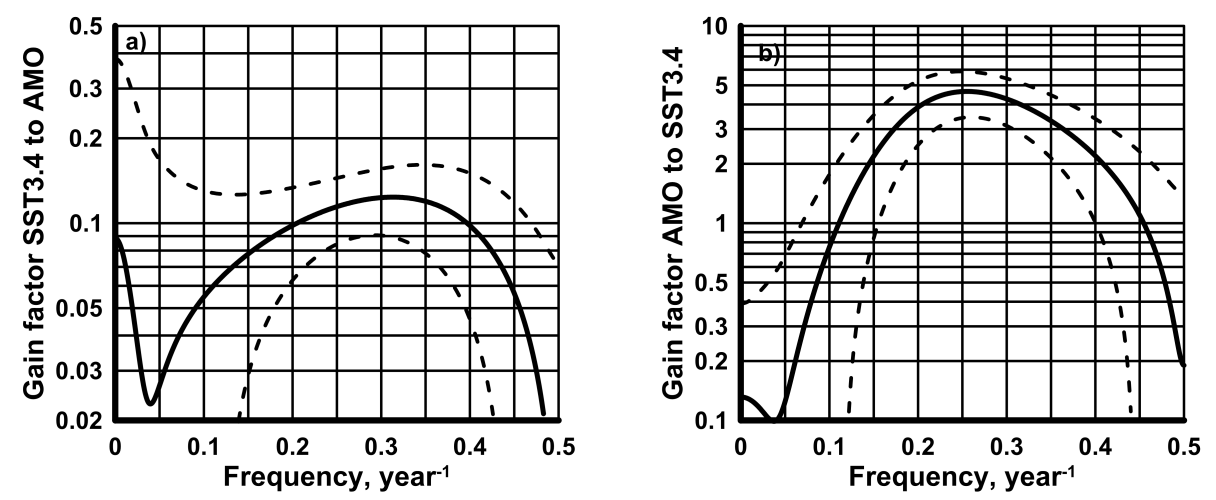

Figure 4. Gain factors SST3.4-AMO (a) and AMO-SST3.4 (b) with approximate $90 \%$ confidence bounds (dashed lines).

and gain factors are shaped similarly because the coherence function is bell-shaped and the AMO and SST3.4 spectra are rather flat at intermediate frequencies.

The phase factors in this case cannot give explicit information about the AMO-SST3.4 system because its feedback loop is closed (interaction between AMO and SST3.4). We cannot compare our spectra with those shown in Park and Dusek (2013) because their spectral estimates are given without confidence bounds but generally the shapes of the spectra at frequencies below $0.5 \mathrm{yr}^{-1}$ seem to be rather similar.

\section{Conclusions and comments}

1. Relations between time series should not be studied on the basis of cross-correlation coefficients and regression equations. An efficient approach within the framework of time series analysis includes two stages both involving parametric (preferably, autoregressive) modeling:

- fitting a stochastic difference equation to the time series (time domain), analyzing the selected model, and

- using the fitted equation to calculate and analyze frequency-domain characteristics (spectra and coherent spectra, coherence function(s), gain and phase factor(s)).

This two-pronged approach is little known in climatology and related sciences.

2. Methods of multivariate time series analysis should be used in all cases, irrespective of the value of the cross-correlation coefficient. The cross-correlation coefficients and regression equation do not generally describe relations between time series. In particular, a low cross-correlation coefficient does not necessarily mean the lack of even a strictly linear dependence between the time series.

3. The stochastic difference equation (Eq. 9) with the innovation sequence covariance matrix (Eq. 10) shows quan- titatively that AMO and SST3.4 interact with each other so that AMO and SST3.4 can be regarded as either inputs or outputs to the AMO-SST3.4 system. It also reveals that the system's memory extends for 2 years. The dependence of AMO and SST3.4 upon their own past and upon the past of the other time series explains about 60 and $25 \%$ of the AMO and SST3.4 variances, respectively.

4. The frequency-domain analysis of the system shows that the spectra of AMO and SST3.4 behave in a different manner, with the AMO spectrum decreasing fast with frequency and with a relatively flat SST3.4 spectrum.

5. In spite of the very low cross-correlation coefficient between the time series of AMO and SST3.4, a close linear dependence between them was shown to exist at intermediate frequencies corresponding to timescales from 3 to 5 years. The coherence between AMO and SST3.4 is statistically significant in a wide frequency band centered at $0.27 \mathrm{yr}^{-1}$ where the coherence peaks at 0.74 . This result has been obtained earlier by Park and Dusek (2013) for a similar climatic system.

6. The coherent spectra AMO-SST3.4 and SST3.4-AMO are statistically significant at frequencies from 0.18 to $0.38 \mathrm{yr}^{-1}$ contributing $25-55 \%$ of the spectral densities of SST3.4 and AMO to each other. The gain factors in the band behave in a manner similar to the behavior of the coherent spectra and peak at about 0.1 and 4.0, respectively.

These conclusions provide answers to the questions formulated in the introduction to this work.

Climatic time series can often be treated as Gaussian. The ability to use a Gaussian approximation is important because for such time series the nonlinear approach cannot give better results than what is obtained within the linear approximation. This latter statement holds, in particular, for time series extrapolation within the framework of the Kolmogorov- 
Wiener theory. Also, as shown by Choi and Cover (1984), the random process that maximizes the entropy rate under constraints on $p$ first values of the correlation function is a Gaussian autoregressive process of order $p$.

Many time series, especially at climatic timescales, are short; that is, their lengths do not exceed the timescales of interest by orders of magnitude. Therefore, the nonparametric analysis in the frequency domain may not be efficient because, with short time series, it would produce less reliable results. Besides, the nonparametric approach does not allow one to obtain explicit stochastic models in the time domain. (These are two more reasons to prefer the parametric modeling.)
A parametric (first of all, autoregressive) analysis in time and frequency domains is effective because it results in relatively accurate estimates due to the postulation of a stochastic model for the time series. In particular, the frequency-domain estimates obtained with the properly selected order satisfy, in the Gaussian case, the requirements of the maximum entropy spectral analysis. However, it is not correct to say that any autoregressive spectral estimate has this important property. The number of parameters to be estimated should always be much smaller than the time series length. If, for example, the length $N \Delta t$ of a bivariate time series is 128 years, one should hardly expect statistically reliable results for models with AR orders higher than 5 (20 AR coefficients plus 3 elements of the innovation sequence covariance matrix plus 23 estimate errors to be estimated). This is one of the reasons why it is not possible to make any realistic conclusions about the presence of 60-70 years long "periods", "cycles", "oscillations", or about any other features at such large timescales unless the time series is at least 300-400 years long (e.g., Gulev et al., 2013). For parametric models, it is strongly recommended to determine the model's order and, consequently, the number of parameters to be estimated, on the basis of order selection criteria; an improper selection of the order invalidates the results of analysis. 


\section{Appendix A}

The following preliminary conclusion was made in Privalsky et al. (1987) on the basis of a Monte Carlo experiment with several autoregressive and one moving-average models with 5000 time series of length $N=50,100$, and 200: "when no sharp peaks appear in the spectral characteristics of a [bivariate] time series, their [autoregressive estimates] seem to be distributed more or less similar to respective [Blackman-Tukey estimates], possess smaller bias and variance and, as a first and conservative approximation, the equivalent number of degrees of freedom of [autoregressive estimates] is $n=N / 2 p$; their approximate confidence bounds can be found in the same manner as for [the] nonparametric spectral estimates (Bendat and Piersol, 1986)." 
Edited by: G. Lohmann

\section{References}

Bendat, J. and Piersol A.: Measurement and Analysis of Random Data, Wiley, New York, 1966.

Bendat, J. and Piersol, A.: Random Data, Analysis and Measurements Procedures, 2nd Edn., Wiley, New York, 1986.

Bendat, J. and Piersol, A.: Random Data, Analysis and Measurements Procedures, 4th Edn., Wiley, Hoboken, 2010.

Box, G. and Jenkins, G.: Time Series Analysis, Forecasting and Control, Holden-Day, San Francisco, 1970.

Box, G., Jenkins, G., and Reinsel, G.: Time Series Analysis, Forecasting and Control, 4th Edn., John Wiley and Sons, Hoboken, NJ, 2008.

Choi, B. and Cover, T.: An information-theoretic proof of Burg's maximum entropy spectrum, Proc. IEEE, 72, 1094-1096, 1984.

Emery, W. and Thomson, R.: Data Analysis Methods in Physical Oceanography, 2nd Edn., Elsevier, Amsterdam, 2004.

Enfield, D., Mestas-Nunez, A., and Trimble, P.: The Atlantic Multidecadal Oscillation and its relationship to rainfall and river flows in the continental U.S., Geophys. Res. Lett., 28, 2077-2080, 2001.

Gelfand, I. and Yaglom, A.: Calculation of the amount of information about a random function contained in another such function, Uspekhi Matematicheskikh Nauk, 12:3-52, 1957, English translation: American Mathematical Society Translation Series 2, 12, 199-246, 1959.

Ghanbari, R., Bravo, H., Magnuson, J., Hyzer, W., and Benson, B.: Coherence between lake ice cover, local climate and teleconnections (Lake Mendota, Wisconsin), J. Hydrol., 374, 282-293, 2009.

Granger, C. W. J.: Investigating causal relations by econometric models and crossspectral methods, Econometrica, 37, 424-438, 1969.

Granger, C. W. J. and Hatanaka, M.: Spectral Analysis of Economic Time Series. Princeton University Press, Princeton, New Jersey, 1964.

Gulev, S. K., Latif, M., Keenlyside, N., Park, W., and Koltermann, K. P.: North Atlantic ocean control on surface heat flux on multidecadal time scales, Nature, 499, 464-467, 2013.

Hannan, E. and Quinn, B.: The determination of the order of an autoregression, J. R. Statist. Soc., B-41, 190-195, 1979.
Morice, C., Kennedy, J., Rayner, N., and Jones, P.: Quantifying uncertainties in global and regional temperature change using an ensemble of observational estimates: the HadCRUT4 dataset, J. Geophys. Res., 117, D08101, doi:10.1029/2011JD017187, 2012.

Oladipo, E. O.: Power spectra and coherence of drought in the Interior Plains, J. Climatol., 7, 477-491, 1987.

Park, J. and Dusek, G.: ENSO components of the Atlantic multidecadal oscillation and their relation to North Atlantic interannual coastal sea level anomalies, Ocean Sci., 9, 535-543, doi:10.5194/os-9-535-2013, 2013.

Parzen, E.: Multiple time series: determining the order of autoregressive approximating schemes, in: Multivariate Analysis IV, North Holland Publishing Company, Amsterdam, 283-295, 1977.

Percival, D. D. and Walden, A. T.: Spectral Analysis for Physical Applications. Multitaper and Conventional Univariate Techniques, Cambridge University Press, 1993.

Philander, S.: El Niño, La Niña, and the Southern Oscillation, Academic Press, San Diego, 1989.

Privalsky, V.: Stochastic models and spectra of interannual variability of mean annual surface temperature in the North Atlantic, Dynam. Atmos. Oceans, 12, 1-18, 1988.

Privalsky, V. and Jensen, D.: Assessment of the influence of ENSO on annual global air temperature, Dynam. Atmos. Oceans, 22, 161-178, 1995.

Privalsky, V. and Muzylev, S.: An Experimental Stochastic Model of the El Niño - Southern Oscillation System at Climatic Time Scales, Univ. J. Geosci., 1, 28-36, 2013.

Privalsky, V., Protsenko, I., and Fogel, G.: The sampling variability of autoregressive spectral estimates for two-variate hydrometeorological processes, Proc. 1st World Congress of the Bernoulli Society on Mathematical Stat. Theory and Applications, Vol. 2, VNU Science Press, Utrecht, 651-654, 1987.

Robinson, E.: Multichannel Time Series Analysis with Digital Computer Programs. Holden-Day, San Francisco, 1967.

Robinson, E. and Treitel, S.: Geophysical Signal Analysis, PrenticeHall, Englewood Cliffs, NJ, 1980.

Schneider, U. and Schönwiese, C.-D.: Some statistical characteristics of El Niño-Southern Oscillation and the North Atlantic Oscillation indices, Atmósfera, 2, 167-180, 1989.

von Storch, H. and Zwiers, F.: Statistical Analysis in Climate Research. Cambridge University Press, Cambidge, 1999. 